\title{
Moment Distributions of Phase Type
}

\author{
Bladt, Mogens; Nielsen, Bo Friis
}

\section{Published in:}

Stochastic Models

Link to article, DOI:

10.1080/15326349.2011.614192

Publication date:

2011

\section{Document Version}

Publisher's PDF, also known as Version of record

Link back to DTU Orbit

\section{Citation (APA):}

Bladt, M., \& Nielsen, B. F. (2011). Moment Distributions of Phase Type. Stochastic Models, 27(4), 651. https://doi.org/10.1080/15326349.2011.614192

\section{General rights}

Copyright and moral rights for the publications made accessible in the public portal are retained by the authors and/or other copyright owners and it is a condition of accessing publications that users recognise and abide by the legal requirements associated with these rights.

- Users may download and print one copy of any publication from the public portal for the purpose of private study or research.

- You may not further distribute the material or use it for any profit-making activity or commercial gain

- You may freely distribute the URL identifying the publication in the public portal

If you believe that this document breaches copyright please contact us providing details, and we will remove access to the work immediately and investigate your claim. 
This article was downloaded by: [DTU Library]

On: 15 December 2011, At: 05:27

Publisher: Taylor \& Francis

Informa Ltd Registered in England and Wales Registered Number: 1072954 Registered office: Mortimer House, 37-41 Mortimer Street, London W1T 3J H, UK

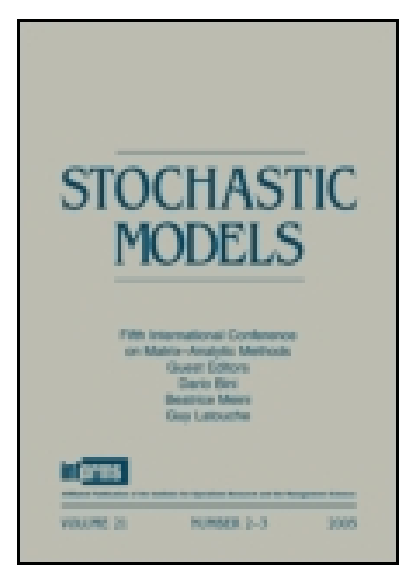

\title{
Stochastic Models
}

Publication details, including instructions for authors and subscription information:

http:// www.tandfonline.com/loi/lstm20

\section{Moment Distributions of Phase Type}

\author{
Mogens Bladt ${ }^{a} \&$ Bo Friis Nielsen ${ }^{b}$ \\ ${ }^{a}$ Instituto de Investigacion en Matematical Aplicados y en Sistemas, Universidad Nacional \\ Autonoma de Mexico, Mexico \\ ${ }^{b}$ Department of Informatics and Mathematical Modelling, Technical University of Denmark, \\ Lyngby, Denmark
}

Available online: 18 Nov 2011

To cite this article: Mogens Bladt \& Bo Friis Nielsen (2011): Moment Distributions of Phase Type, Stochastic Models, 27:4, 651-663

To link to this article: http:// dx.doi.org/ 10.1080/ 15326349.2011.614192

\section{PLEASE SCROLL DOWN FOR ARTICLE}

Full terms and conditions of use: http://www.tandfonline.com/page/terms-and-conditions

This article may be used for research, teaching, and private study purposes. Any substantial or systematic reproduction, redistribution, reselling, loan, sub-licensing, systematic supply, or distribution in any form to anyone is expressly forbidden.

The publisher does not give any warranty express or implied or make any representation that the contents will be complete or accurate or up to date. The accuracy of any instructions, formulae, and drug doses should be independently verified with primary sources. The publisher shall not be liable for any loss, actions, claims, proceedings, demand, or costs or damages whatsoever or howsoever caused arising directly or indirectly in connection with or arising out of the use of this material. 


\title{
MOMENT DISTRIBUTIONS OF PHASE TYPE
}

\author{
Mogens Bladt ${ }^{1}$ and Bo Friis Nielsen ${ }^{2}$ \\ ${ }^{1}$ Instituto de Investigacion en Matematical Aplicados y en Sistemas, \\ Universidad Nacional Autonoma de Mexico, Mexico \\ ${ }^{2}$ Department of Informatics and Mathematical Modelling, Technical University \\ of Denmark, Lyngby, Denmark
}

\begin{abstract}
$\square \quad$ Moment distributions of phase-type and matrix-exponential distributions are shown to remain within their respective classes. We provide a probabilistic phase-type representation for the former case and an alternative representation, with an analytically appealing form, for the latter. First order moment distributions are of special interest in areas like demography and economics, and we calculate explicit formulas for the Lorenz curve and Gini index used in these disciplines.
\end{abstract}

Keywords Gini index; Lorenz curve; Matrix-exponential distributions; Moment distribution; Phase-type distributions; Size-biasing; Waiting time paradox.

Mathematics Subject Classification Primary 60J27, 60J75; Secondary 60K05.

\section{INTRODUCTION}

In this article, we consider moment distributions obtained from distributions which are either of phase-type or matrix-exponential. We shall prove that if the underlying distribution is of phase-type, then the corresponding moment distribution of any order is also phase-type distributed. If the underlying distribution is a matrix-exponential distribution, then moment distributions of any order are again matrixexponential. For the phase-type case we shall provide two phase-type representations of the moment distributions, whereas for the matrixexponential case we shall provide an alternative representation with an appealing structure which in general will not be of phase-type.

Received December 2010; Accepted August 2011

Address correspondence to Mogens Bladt, Institute for Applied Mathematics and Systems, National University of Mexico, A.P. 20-726, Mexico City 01000, Mexico; E-mail: bladt@stats.iimas.unam.mx 
Moment distributions are important in various fields of application such as demography, economics, engineering and geology. They are often referred to as distributions obtained by length biased (first order moment distributions) or size-biased sampling. A well known application is concerning the income distribution in a society. If one samples a dollar, then there is an indication of inequality in the society if the probability that the dollar belongs to a rich person is very different (much higher) than the relative frequency of rich people. The situation is similar to the inspection paradox from renewal theory. For the first order distribution we present an explicit formula for the related Lorenz curve and Gini index, which is a frequently used measure of inequality.

The article is organized as follows. In Section 2 we provide the necessary background and notation on moment distributions and renewal processes. In Section 3 we prove our main results and in Section 4 we calculate the Lorenz curve and Gini index.

\section{BACKGROUND AND NOTATION}

Consider the density function $f$ of a non-negative random variable $X$, then

$$
f_{i}(x)=\frac{x^{i} f(x)}{\mu_{i}}, \quad \text { where } \mu_{i}=\int_{0}^{\infty} x^{i} f(x) d x
$$

are densities of some non-negative random variables $X^{(i)}$ provided that $\mu_{i}$ exists. We say that $f_{i}$ is the density of the $i$ th order moment distribution of $f$. Log-normal, Pareto, and gamma distributions are closed under the formation of moment distributions.

A stationary renewal process with inter-arrival time distribution given by the density $f$ is a delayed renewal process, where the time until the first arrival has density $f_{e}(x)=(1-F(x)) / \mu_{1}=\bar{F}(x) / \mu_{1}$. Here $F$ and $\bar{F}$ are the distribution and survival functions corresponding to $f$. Let $A_{t}$ denote the age of the process at time $t$, i.e., the time from the last arrival to $t$ and $R_{t}$ the residual life-time, which is the time from $t$ until the next arrival. Then the joint distribution of $A_{t}$ and $R_{t}$ is given by (see, e.g., Ref. ${ }^{[9]}$, Proposition 3.2) $\mathbb{P}\left(A_{t}>x, R_{t}>y\right)=\bar{F}_{e}(x+y)$, or equivalently $f_{\left(A_{t}, R_{t}\right)}(x, y)=\mu_{1}^{-1} f(x+y)$. The spread $S_{t}=A_{t}+R_{t}$ is the length of the inter-arrival interval which contains the time point $t$ and is then calculated to have density

$$
f_{S_{t}}(x)=\int_{0}^{x} f_{\left(A_{t}, R_{t}\right)}(x-t, t) d t=\frac{x f(x)}{\mu_{1}},
$$

which is exactly the first order moment distribution of $f$. 
Size biasing in dimensions two and three occur in a similar fashion. Consider a map of a country which is partitioned into counties. We could be interested in estimating the distribution of the county sizes. Sampling counties through realizing a two dimensional Poisson process over the map results in an area biased distribution, where the probability of choosing a county is proportional to its area. Three dimensional size-biased distributions occur when estimating grain sizes by using sieves of increasing fineness and then using the weight of the different groups, which is approximately proportional to the cube of the grain diameters; see, e.g., Ref. ${ }^{[10]}$.

A distribution of a non-negative random variable is called matrixexponential if it has a density function $f$ on the form $f(x)=\alpha \exp (\mathbf{S} x) \mathbf{s}$, where $\alpha$ and $\mathbf{s}$ are row and column vectors respectively and $\mathbf{S}$ is a matrix. The triple $(\boldsymbol{\alpha}, \mathbf{S}, \mathbf{s})$ is called a representation of the matrix-exponential distribution. With e being a column vector of ones, a representation can always be chosen such that $\mathbf{s}=-$ Se and $0 \leq \boldsymbol{\alpha e} \leq 1$. The common dimension $p$ is referred to as the order of the matrix-exponential representation. The order is called minimal if it is not possible to find another representation of $f$ with a strictly lower order, in which case it is called the order of the distribution.

A special sub-class of matrix-exponential distributions are the so-called phase-type distributions. They are defined as the distributions of the times until absorption of Markov jump processes with a finite number of transient states and one absorbing state. In this case, the representations $(\boldsymbol{\alpha}, \mathbf{S}, \mathbf{s})$ may be taken to have probabilistic interpretations as respectively $\boldsymbol{\alpha}$ the initial distribution of the underlying Markov jump process generating the phase-type distribution, $\mathbf{S}$ a sub-intensity matrix of transition rates between the transient states, and $\mathbf{s}$ the exit rate vector of intensities for jumping to an absorbing state.

The $n$th moment of a matrix exponential distribution with representation $(\boldsymbol{\alpha}, \mathbf{S}, \mathbf{s})$ is given by $(-1)^{n+1} n ! \boldsymbol{\alpha} \mathbf{S}^{-(n+1)} \mathbf{s}$. If $\mathbf{s}=-\mathbf{S e}$, which is true for phase-type representations, the formula for the $n$th moment reduces to $n ! \boldsymbol{\alpha}(-\mathbf{S})^{-n} \mathbf{e}$. The cumulative distribution functions are, respectively, given by $1+\alpha \exp (\mathbf{S} x) \mathbf{S}^{-1} \mathbf{s}$ and $1-\alpha \exp (\mathbf{S} x)$ e. For more details on matrixexponential and phase-type distributions we refer to Refs. ${ }^{[2,3,7]}$.

Representations of matrix-exponential and phase-type distributions are not unique. If for example $(\boldsymbol{\alpha}, \mathbf{S}, \mathbf{s})$ is a representation of a matrix-exponential distribution and $\mathbf{M}$ is a non-singular matrix, then $\left(\alpha \mathbf{M}^{-1}, \mathbf{M S M} \mathbf{M}^{-1}, \mathbf{M s}\right)$ is another representation of the same distribution. Of special interest is the case where $\mathbf{M}=\boldsymbol{\Delta}(\mathbf{m})$ is a diagonal matrix with $\mathbf{m}=\left(m_{1}, m_{2}, \ldots, m_{p}\right)$ and $m_{i} \neq 0$ for all $i$ in which case we may construct the time-reversed (or dual) representation by letting $\mathbf{m}=-\alpha \mathbf{S}^{-1}$ for a phase-type representation $(\boldsymbol{\alpha}, \mathbf{S})$. The time reversed representation $(\hat{\alpha}, \widehat{\mathbf{S}})$ is then given by $\hat{\alpha}=\mathbf{s}^{\prime} \mathbf{M}$ and $\widehat{\mathbf{S}}=\mathbf{M}^{-1} \mathbf{S}^{\prime} \mathbf{M}$. For more details, see Refs. ${ }^{[1,4,8]}$. 
Consider a delayed renewal process, where the distribution of the time until the first arrival is matrix-exponentially distributed with representation $(\boldsymbol{\beta}, \mathbf{S}, \mathbf{s})$ and the remaining inter-arrivals are matrixexponentially distributed with common representation $(\alpha, \mathbf{S}, \mathbf{s})$. Then the renewal density is given by $u(x)=\boldsymbol{\beta} \exp ((\mathbf{S}+\mathbf{s} \boldsymbol{\alpha}) x) \mathbf{s}$ and the residual lifetime $R_{t}$ is matrix-exponentially distributed with representation $(\boldsymbol{\beta} \exp ((\mathbf{S}+$ $\mathbf{s} \boldsymbol{\alpha}) t$ ), $\mathbf{S}, \mathbf{s}$ ) (see Ref. ${ }^{[2]}$ for details and further properties). The renewal process is stationary if the delay is matrix-exponentially distributed with representation $\left(-\alpha \mathbf{S}^{-1} / \boldsymbol{\alpha} \mathbf{S}^{-2} \mathbf{s}, \mathbf{S}, \mathbf{s}\right)$.

\section{MOMENT DISTRIBUTIONS OF MATRIX-EXPONENTIAL DISTRIBUTIONS}

Let $f$ be the density of a matrix-exponential distribution with representation $(\alpha, \mathbf{S}, \mathbf{s})$. Then it is clear that any moment distribution $f_{i}$ is again a matrix-exponential distribution. This follows directly from

$$
\frac{d^{i}}{d s^{i}} \hat{f}(s)=\int_{0}^{\infty}(-1)^{i} x^{i} e^{-s x} f(x) d x=(-1)^{i} \mu_{i} \hat{f}_{i}(s) .
$$

Since $\hat{f}(s)$ is a rational function, so is its $i$ th derivative, and hence $\hat{f}_{i}(s)$ is also a rational function which, in turn, is equivalent to $f_{i}$ being matrixexponential. We next address the problem of finding representations for such distributions.

It is often an advantage, in particular in applications of non-linear models, that a matrix-exponential distribution with representation $(\boldsymbol{\alpha}, \mathbf{S}, \mathbf{s})$ satisfies $\mathbf{s}=-$ Se and $0 \leq \boldsymbol{\alpha e} \leq 1$; see Ref. ${ }^{[3]}$. In the following we shall restrict attention to matrix-exponential representations satisfying these two properties.

Theorem 3.1. Consider a matrix-exponential distribution with representation $(\boldsymbol{\alpha}, \mathbf{S}, \mathbf{s})$ such that $\mathbf{s}=-\mathbf{S e}$ and $0 \leq \boldsymbol{\alpha e} \leq 1$. Then its $n$th moment distribution is also matrix-exponential with representation $\left(\boldsymbol{\alpha}_{n}, \mathbf{S}_{n}, \mathbf{s}_{n}\right)$, where

$$
\boldsymbol{\alpha}_{n}=\left(\frac{\alpha \mathbf{S}^{-n}}{\alpha \mathbf{S}^{-n} \mathbf{e}}, \mathbf{0}, \ldots, \mathbf{0}\right) \quad \mathbf{S}_{n}=\left(\begin{array}{ccccc}
\mathbf{S} & -\mathbf{S} & \mathbf{0} & \ldots & \mathbf{0} \\
\mathbf{0} & \mathbf{S} & -\mathbf{S} & \ldots & \mathbf{0} \\
\ldots & \ldots & \ldots & \ldots & \ldots \\
\mathbf{0} & \mathbf{0} & \mathbf{0} & \mathbf{0} & \mathbf{S}
\end{array}\right), \quad \mathbf{s}_{n}=\left(\begin{array}{c}
0 \\
0 \\
. . \\
\mathbf{s}
\end{array}\right) .
$$

Proof. When calculating $\boldsymbol{\alpha}_{n} \exp \left(\mathbf{S}_{n} x\right) \mathbf{s}_{n}$ we see that only the upper right term is of importance in the matrix-exponential. This term is calculated to be $\left((-1)^{n} / n !\right) x^{n} \mathbf{S}^{n} \exp (\mathbf{S} x)$ by taking powers of $\widehat{\mathbf{S}}$ and summing up. Thus

$$
\boldsymbol{\alpha}_{n} e^{\mathbf{S}_{n x}} \mathbf{s}_{n}=\frac{\boldsymbol{\alpha} \mathbf{S}^{-n}}{\boldsymbol{\alpha} \mathbf{S}^{-n} \mathbf{e}} \frac{(-1)^{n}}{n !} x^{n} \mathbf{S}^{n} e^{\mathbf{S}_{x}} \mathbf{s}=\frac{x^{n} \boldsymbol{\alpha} e^{\mathbf{S}_{x}} \mathbf{S}}{(-1)^{n} n ! \boldsymbol{\alpha} \mathbf{S}^{-n} \mathbf{e}}=\frac{x^{n} \boldsymbol{\alpha} e^{\mathbf{S}_{x}} \mathbf{s}}{\mu_{n}},
$$


where $\mu_{n}$ (as usual) denotes the $n$th moment of the matrix-exponential distribution.

Corollary 3.2. A similar calculation yields the following

$$
F_{n}(x)=1-\frac{\boldsymbol{\alpha} \mathbf{S}^{-n}}{\boldsymbol{\alpha} \mathbf{S}^{-n} \mathbf{e}} \sum_{i=0}^{n} \frac{(-x \mathbf{S})^{i}}{i !} e^{\mathbf{S} x} \mathbf{e}
$$

The $n$th order moment distributions of an exponential distribution with intensity $\lambda$ is an $\operatorname{Erlang}_{n+1}(\lambda)$ distribution. Theorem 3.1 and Corollary 3.2 are nice examples of the generalization of the exponential distribution to matrix-exponential distributions. As $\left(\left(\alpha \mathbf{S}^{-1} \mathbf{e}\right)^{-1} \boldsymbol{\alpha} \mathbf{S}^{-1}, \mathbf{S}, \mathbf{s}\right)$ is a representation for a matrix-exponential distribution we see that also $\left(\left(\alpha \mathbf{S}^{-n} \mathbf{e}\right)^{-1} \alpha \mathbf{S}^{-n}, \mathbf{S}, \mathbf{s}\right)$ is a representation for a matrixexponential distribution for all non-negative integer $n$, implying that $\left(\left(\boldsymbol{\alpha}(-\mathbf{S})^{i} e^{\mathbf{S}_{x}} \mathbf{e}\right)^{-1} \boldsymbol{\alpha}(-\mathbf{S})^{i} e^{\mathbf{S} x}, \mathbf{S}, \mathbf{s}\right)$ is a representation for a matrix-exponential distribution for all non-negative integer $i$ and non-negative $x$. It follows that the $n$th order moment distribution can be interpreted as the distribution of a sum of $n$ identically distributed matrix-exponentially distributed random variables, exactly as for the exponential distribution, although the individual random variables are dependent in the matrixexponential case. The representation for each of the individual variables can be chosen as $\left(\left(\alpha \mathbf{S}^{-n} \mathbf{e}\right)^{-1} \alpha \mathbf{S}^{-n}, \mathbf{S}, \mathbf{s}\right)$.

If the underlying distribution is a phase-type distribution with representation $(\boldsymbol{\alpha}, \mathbf{S})$, then the above representation for the $n$th order moment distribution will only yield a phase-type representation when the distribution is exponential or hyper-exponential, since otherwise there will be negative off-diagonal elements present. In the case of phase-type distributions, the above representation will therefore be of limited use.

We still need to prove that the $n$th order moment distribution of a phase-type distribution is of phase-type. To this end it is sufficient to prove that the first order moment distribution is of phase-type as the $n$th order can be obtained by an iterative argument.

Theorem 3.3. Consider a phase-type distribution with representation $(\boldsymbol{\alpha}, \mathbf{S})$. Then the first moment distribution is again of phase-type. A representation is $\left(\hat{\boldsymbol{\alpha}}_{1}, \widehat{\mathbf{S}}_{1}\right)$, where

$$
\hat{\boldsymbol{\alpha}}_{1}=\left(\mathbf{s}^{\prime} \boldsymbol{\Delta}\left(\mathbf{m}_{2}\right), \mathbf{0}\right), \quad \widehat{\mathbf{S}}_{1}=\left(\begin{array}{cc}
\boldsymbol{\Delta}^{-1}\left(\mathbf{m}_{2}\right) \mathbf{S}^{\prime} \Delta\left(\mathbf{m}_{2}\right) & \rho_{1}^{-1} \boldsymbol{\Delta}^{-1}\left(\mathbf{m}_{2}\right) \boldsymbol{\Delta}\left(\mathbf{m}_{1}\right) \\
\mathbf{0} & \boldsymbol{\Delta}^{-1}\left(\mathbf{m}_{1}\right) \mathbf{S}^{\prime} \Delta\left(\mathbf{m}_{1}\right)
\end{array}\right),
$$

with $\rho_{i}=\alpha\left(-\mathbf{S}^{-i}\right) \mathbf{e}$ and $\mathbf{m}_{i}=\rho_{i-1}^{-1} \boldsymbol{\alpha}(-\mathbf{S})^{-i}$. 
Proof. Consider a stationary renewal process with an inter-arrival time distribution which is of phase-type with representation $(\boldsymbol{\alpha}, \mathbf{S})$. Then the distribution of the phases by time $t$ is $\pi_{1}=\rho_{1}^{-1} \mathbf{m}_{1}$, with $\rho_{1}=\mathbf{m}_{1} \mathbf{e}$. Hence both $R_{t}$ and $A_{t}$ are phase-type distributed with representation $\left(\boldsymbol{\pi}_{1}, \mathbf{S}\right)$ or $\left(\pi_{1}, \widehat{\mathbf{S}}\right)$ with $\widehat{\mathbf{S}}=\boldsymbol{\Delta}^{-1}\left(\mathbf{m}_{1}\right) \mathbf{S}^{\prime} \boldsymbol{\Delta}\left(\mathbf{m}_{1}\right)$. The distribution of the spread, $A_{t}+R_{t}$, is the first order moment distribution of the phase-type distribution with representation $(\boldsymbol{\alpha}, \mathbf{S})$. A representation of the spread can be established using a phase-type argument as follows. The development is illustrated in Figure 1. The phase at $t$ of the Markov jump process is the phase of $A_{t}$ immediately before absorption and the initial phase of $R_{t}$. To find the distribution of $A_{t}$ we realize that the time to the initial point of the interval is found by running the phase-type renewal process in reversed time, such that a representation of $\widehat{A}_{t}$ is $\left(\pi_{1}, \widehat{\mathbf{S}}\right)$. To obtain a phase type representation for the spread we must ensure that the initial phase of $R_{t}$ is the same as the phase of $A_{t}$ immediately before absorption, or when considering the process in reversed time, that the initial phase of $\widehat{A}_{t}$ is the phase of $\widehat{R}_{t}$ immediately before absorption. To complete the argument we need the time reversed representation of $\left(\pi_{1}, \mathbf{S}\right)$. Here $\mathbf{m}=-\pi_{1} \mathbf{S}^{-1}=\mathbf{m}_{2}$ and the time reversed representation for $R_{t}$ (the representation for $\widehat{R}_{t}$ ) is

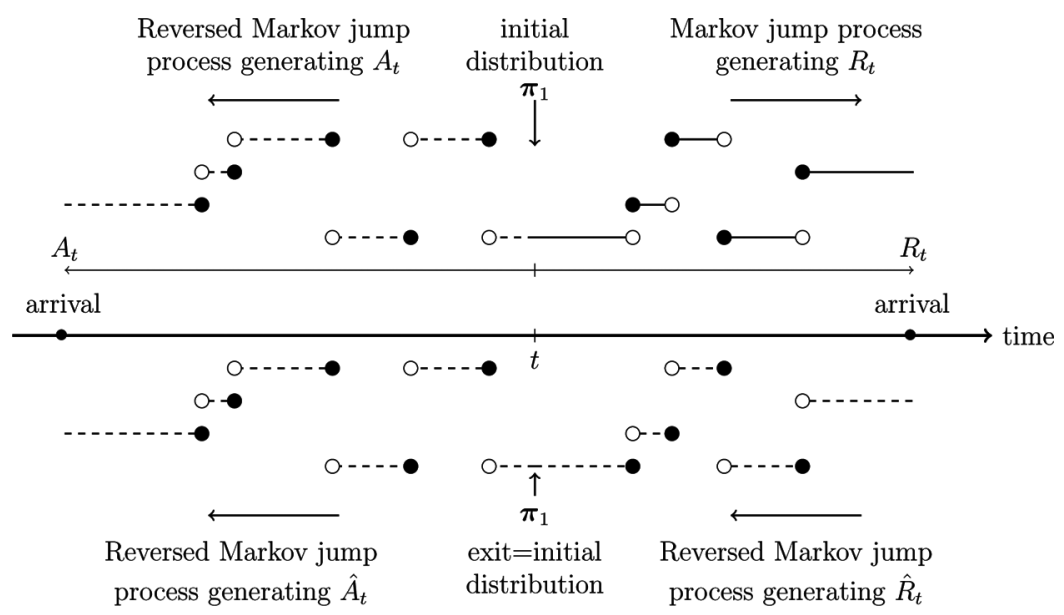

FIGURE 1 At time $t$ the distribution of states in the stationary PH renewal process is $\pi_{1} . A_{t}$ and $R_{t}$ can be generated by initiating according to $\pi_{1}$ in the same phase as shown above the time axis and running them in each their direction. The dashed trajectory corresponds to the $A_{t}$ process running reverse in time and the continuous lines corresponds to the $R_{t}$ process running forward in time. Under the time axis is a realization of the time-reversed Markov jump process generating the reversed versions $\widehat{R}_{t}$ and $\widehat{A}_{t}$ of $R_{t}$ and $A_{t}$. The transition from the upper to the lower block corresponds to the inspection time " $\mathrm{t}$ " and the phase of the underlying process is not changed, while the marginal distribution of phases at the time of change from the process generating $\widehat{R}_{t}$ to the process generating $\widehat{A}_{t}$ is $\pi_{1}$. 
$\left(\mathbf{s}^{\prime} \Delta\left(\mathbf{m}_{2}\right), \Delta^{-1}\left(\mathbf{m}_{2}\right) \mathbf{S}^{\prime} \Delta\left(\mathbf{m}_{2}\right)\right)$, with exit vector

$$
-\Delta^{-1}\left(\mathbf{m}_{2}\right) \mathbf{S}^{\prime} \Delta\left(\mathbf{m}_{2}\right) \mathbf{e}=-\Delta^{-1}\left(\mathbf{m}_{2}\right) \rho_{1}^{-1}\left(\boldsymbol{\alpha}(-\mathbf{S})^{-2} \mathbf{S}\right)^{\prime}=\rho_{1}^{-1} \Delta^{-1}\left(\mathbf{m}_{2}\right) \mathbf{m}_{1}^{\prime} .
$$

Now when the process of $\widehat{R}_{t}$ terminates, the process for the spread resumes in reversed time according to the standard reversed generator $\widehat{\mathbf{S}}$ to account for the $A_{t}$ part of the spread, so the block matrix leading from the states representing $\widehat{R}_{t}$ to the states representing $\widehat{A}_{t}$ is a diagonal matrix of the vector given by Eq. (1).

As the representation of Theorem 3.3 and its proof is motivated by a time reversal argument it is tempting to investigate the corresponding time reversed representation. One would think that there should be such a forward representation where the lower order block would be simply $\mathbf{S}$ and where the initial distribution into that block would be $\pi_{1}$ to ensure the correct marginal distribution of $R_{t}$.

Corollary 3.4. $\quad$ Consider a phase-type distribution with representation $(\boldsymbol{\alpha}, \mathbf{S})$. Its first order moment distribution can be represented by a phase-type representation $\left(\boldsymbol{\alpha}_{1}, \mathbf{S}_{1}\right)$ with

$$
\boldsymbol{\alpha}_{1}=\left(\rho_{1}^{-1} \boldsymbol{\alpha} \boldsymbol{\Delta}\left(\boldsymbol{\rho}_{1}\right), \mathbf{0}\right), \mathbf{S}_{1}=\left(\begin{array}{cc}
\boldsymbol{\Delta}^{-1}\left(\boldsymbol{\rho}_{1}\right) \mathbf{S} \boldsymbol{\Delta}\left(\boldsymbol{\rho}_{1}\right) \boldsymbol{\Delta}^{-1}\left(\boldsymbol{\rho}_{1}\right) \\
\mathbf{0} & \mathbf{S}
\end{array}\right), \quad \text { where } \boldsymbol{\rho}_{1}=(-\mathbf{S})^{-1} \mathbf{e}
$$

Proof. The time reversed representation is found by calculating $\mathbf{m}=\hat{\boldsymbol{\alpha}}(-\widehat{\mathbf{S}})^{-1}, \boldsymbol{\Delta}^{-1}(\mathbf{m}) \widehat{\mathbf{S}}^{\prime} \boldsymbol{\Delta}(\mathbf{m})$, and $(-\widehat{\mathbf{S e}})^{\prime} \boldsymbol{\Delta}(\mathbf{m})$. By reordering the state space such that the phases related to $A_{t}$ precede those related to $R_{t}$ we get the formulation of the corollary.

The $i$ th element of the initial vector $\rho_{1}^{-1} \boldsymbol{\alpha} \Delta\left(\rho_{1}\right)$ is the proportional contribution to the mean from trajectories initiated in state $i$, and the $i$ th diagonal element of the block transition matrix $\Delta^{-1}\left(\rho_{1}\right)$ is the reciprocal of that same mean and can be interpreted as the rate of absorption from phase $i$. The interpretation of the $(i, j)$ th off-diagonal element of the sub-generator $\boldsymbol{\Delta}^{-1}\left(\boldsymbol{\rho}_{1}\right) \mathbf{S} \boldsymbol{\Delta}\left(\boldsymbol{\rho}_{1}\right)$ is the original transition rate $S_{i j}$ multiplied with the ratio of the mean time before absorption of trajectories initiated in state $j$ and $i$, respectively. Thus in the forward representation the generator, somewhat surprisingly, does not depend on the initial distribution, so the generator is the same for all moment distributions of phase-type distributions with the sub-generator $\mathbf{S}$. As an example of Corollary 3.4 we have that the $\left(\boldsymbol{\alpha}_{1}, \mathbf{S}_{1}\right)$ representation for an $\operatorname{Erlang}_{2}(\lambda)$ 
distribution becomes

$$
\left.(1,0,0,0),\left(\begin{array}{cccc}
-\lambda & \frac{1}{2} \lambda & \frac{1}{2} \lambda & 0 \\
0 & -\lambda & 0 & \lambda \\
0 & 0 & -\lambda & \lambda \\
0 & 0 & 0 & -\lambda
\end{array}\right)\right) .
$$

As the first order moment distribution of an $\operatorname{Erlang}_{2}(\lambda)$ distribution is an $\operatorname{Erlang}_{3}(\lambda)$ distribution the $\left(\boldsymbol{\alpha}_{1}, \mathbf{S}_{1}\right)$ representation is not minimal. Nevertheless, that representation gives some probabilistic insight. The transition from the upper to the lower block corresponds to the time " $t$ " of inspection. The probability that inspection will occur in each of the two phases is $\frac{1}{2}$. We have failed, to make a direct probabilistic argument that leads to the representation $\left(\boldsymbol{\alpha}_{1}, \mathbf{S}_{1}\right)$ in general. By considering the case of a hyper-exponential distribution one sees that the dimension of the $\left(\boldsymbol{\alpha}_{1}, \mathbf{S}_{1}\right)$ representation can not always be reduced. The distribution of the spread for a phase-type renewal process was discussed in Ref. ${ }^{[6]}$ but neither the phase-type property nor its representation was given there.

Since the second order moment distribution can be obtained as the first order moment distribution of the first order moment distribution, then by applying Theorem 3.3 to the phase-type representation $\left(\hat{\boldsymbol{\alpha}}_{1}, \widehat{\mathbf{S}}_{1}\right)$ we see that the second order moment distribution is again of phase-type. If the order of $(\boldsymbol{\alpha}, \mathbf{S})$ is $p$, then the order of the first order moment distribution is $2 p$ and the order of the second order moment distribution $4 p$. Proceeding in this way, we see that the $n$th order moment distribution is of phase-type of order $2^{n} p$. We now provide an alternative phase-type representation for the $n$th order moment distribution of order $(n+1) p$. The representation provided coincides with the representation of Theorem 3.3 for first order moment distributions.

Theorem 3.5. Consider a phase-type distribution with representation $(\boldsymbol{\alpha}, \mathbf{S})$. Then the nth order moment distribution is again of phase-type with representation $\left(\hat{\boldsymbol{\alpha}}_{n}, \widehat{\mathbf{S}}_{n}\right)$, where

$$
\begin{aligned}
\hat{\boldsymbol{\alpha}}_{n}= & \left(\frac{\rho_{n+1}}{\rho_{n}} \mathbf{s}^{\prime} \boldsymbol{\Delta}\left(\boldsymbol{\pi}_{n+1}\right), \mathbf{0}, \ldots, \mathbf{0}\right) \\
\widehat{\mathbf{S}}_{n}= & {\left[\begin{array}{cccccc}
\mathbf{C}_{n+1} & \mathbf{D}_{n+1} & \mathbf{0} & \ldots & \mathbf{0} & \mathbf{0} \\
\mathbf{0} & \mathbf{C}_{n} & \mathbf{D}_{n} & \ldots & \mathbf{0} & \mathbf{0} \\
\mathbf{0} & \mathbf{0} & \mathbf{C}_{n-1} & \ldots & \mathbf{0} & \mathbf{0} \\
\vdots & \vdots & \vdots & \ldots: & \vdots & \vdots \\
\mathbf{0} & \mathbf{0} & \mathbf{0} & \ldots & \mathbf{C}_{2} & \mathbf{D}_{2} \\
\mathbf{0} & \mathbf{0} & \mathbf{0} & \ldots & \mathbf{0} & \mathbf{C}_{1}
\end{array}\right] }
\end{aligned}
$$


and $\rho_{i}=\mu_{i} / i !=\alpha(-\mathbf{S})^{-i} \mathbf{e}$ are the reduced moments,

$$
\boldsymbol{\pi}_{i}=\rho_{i}^{-1} \boldsymbol{\alpha}(-\mathbf{S})^{-i}, \quad \mathbf{C}_{i}=\boldsymbol{\Delta}\left(\boldsymbol{\pi}_{i}\right)^{-1} \mathbf{S}^{\prime} \Delta\left(\boldsymbol{\pi}_{i}\right) \quad \text { and } \quad \mathbf{D}_{i}=\frac{\rho_{i-1}}{\rho_{i}} \boldsymbol{\Delta}\left(\boldsymbol{\pi}_{i}\right)^{-1} \boldsymbol{\Delta}\left(\boldsymbol{\pi}_{i-1}\right) .
$$

Proof. We lack a probabilistic argument so the proof is purely analytical. We prove that the Laplace transform corresponding to $\left(\boldsymbol{\alpha}_{n}, \mathbf{S}_{n}\right)$ is identical to the one of Theorem 3.1. Noticing that $\left(u \mathbf{I}-\boldsymbol{\Delta}^{-1} \mathbf{S}^{\prime} \boldsymbol{\Delta}\right)^{-1}=\boldsymbol{\Delta}^{-1}(u \mathbf{I}-$ $\left.\mathbf{S}^{\prime}\right)^{-1} \Delta$ it is readily seen that the Laplace transform $L(u)$ corresponding to $\left(\boldsymbol{\alpha}_{n}, \mathbf{S}_{n}\right)$ amounts to

$$
\begin{aligned}
L(u)= & \left(\frac{\rho_{n+1}}{\rho_{n}} \mathbf{s}^{\prime} \Delta\left(\boldsymbol{\pi}_{n+1}\right)\right) \\
& \times\left[\prod_{i=0}^{n-1}\left(u \mathbf{I}-\boldsymbol{\Delta}\left(\boldsymbol{\pi}_{n+1-i}\right)^{-1} \mathbf{S}^{\prime} \Delta\left(\boldsymbol{\pi}_{n+1-i}\right)\right)^{-1} \Delta\left(\boldsymbol{\pi}_{n+1-i}\right)^{-1} \Delta\left(\boldsymbol{\pi}_{n-i}\right) \frac{\rho_{n-i}}{\rho_{n+1-i}}\right] \\
& \times\left(u \mathbf{I}-\boldsymbol{\Delta}\left(\boldsymbol{\pi}_{1}\right)^{-1} \mathbf{S}^{\prime} \boldsymbol{\Delta}_{1}\right)^{-1} \Delta\left(\boldsymbol{\pi}_{1}\right)^{-1} \boldsymbol{\pi}_{0}^{\prime} \frac{1}{\rho_{1}} \\
= & \rho_{n}^{-1} \mathbf{S}^{\prime}\left(u \mathbf{I}-\mathbf{S}^{\prime}\right)^{-n-1} \boldsymbol{\alpha}^{\prime} \\
= & \rho_{n}^{-1} \boldsymbol{\alpha}(u \mathbf{I}-\mathbf{S})^{-(n+1)} \mathbf{s} \\
= & \boldsymbol{\pi}_{n}\left[(u \mathbf{I}-\mathbf{S})^{-1}(-\mathbf{S})\right]^{n}(u \mathbf{I}-\mathbf{S})^{-1} \mathbf{s}
\end{aligned}
$$

coinciding with the Laplace transform of Theorem 3.1.

The forward representation generalized from Corollary 3.4 is

Corollary 3.6. The nth order moment distribution of a phase-type distribution with representation $(\boldsymbol{\alpha}, \mathbf{S})$ has a phase-type representation $\left(\boldsymbol{\alpha}_{n}, \mathbf{S}_{n}\right)$ with

$$
\begin{aligned}
& \boldsymbol{\alpha}_{n}=\left(\rho_{n}^{-1} \boldsymbol{\alpha \Delta}\left(\boldsymbol{\rho}_{n}\right), \mathbf{0}, \mathbf{0}, \ldots, \mathbf{0}\right) \\
& \mathbf{S}_{n}=\left(\begin{array}{ccccc}
\boldsymbol{\Delta}^{-1}\left(\boldsymbol{\rho}_{n}\right) \mathbf{S} \boldsymbol{\Delta}\left(\boldsymbol{\rho}_{n}\right) & \boldsymbol{\Delta}^{-1}\left(\boldsymbol{\rho}_{n}\right) \boldsymbol{\Delta}\left(\boldsymbol{\rho}_{n-1}\right) & \mathbf{0} & \ldots & \mathbf{0} \\
\mathbf{0} & \boldsymbol{\Delta}^{-1}\left(\boldsymbol{\rho}_{n-1}\right) \mathbf{S} \boldsymbol{\Delta}\left(\boldsymbol{\rho}_{n-1}\right) & \boldsymbol{\Delta}^{-1}\left(\boldsymbol{\rho}_{n-1}\right) \boldsymbol{\Delta}\left(\boldsymbol{\rho}_{n-2}\right) & \ldots & \mathbf{0} \\
\mathbf{0} & \mathbf{0} & \boldsymbol{\Delta}^{-1}\left(\boldsymbol{\rho}_{n-2}\right) \mathbf{S} \boldsymbol{\Delta}\left(\boldsymbol{\rho}_{n-2}\right) & \ldots & \mathbf{0} \\
\vdots & \vdots & \vdots & \ldots: & \vdots \\
\mathbf{0} & \mathbf{0} & \mathbf{0} & \ldots & \mathbf{S}
\end{array}\right),
\end{aligned}
$$

with $\boldsymbol{\rho}_{n}=(-\mathbf{S})^{-n} \mathbf{e}$ and $\rho_{n}=\alpha \boldsymbol{\rho}_{n}$

Proof. The proof technique is similar to that of Corollary 3.4. 


\section{LORENZ CURVE AND THE GINI INDEX}

If $F$ is a distribution function and $F_{1}$ the distribution function of the corresponding first order moment distribution, then the parametric curve $\gamma: t \rightarrow\left(F(t), F_{1}(t)\right), t \in[0, \infty)$ is called the Lorenz curve. Traditionally the Lorenz curve is used to illustrate inequality in a society with the interpretation that the poorest $x=F(t)$ per cent of the population posses $y=F_{1}(t)$ per cent of the total wealth. Another measure frequently used is the Gini index, which is defined as twice the area between the curve $\gamma$ and the line $y=x$. It compares the area between the curve $\gamma$ and the line $y=x$ to the area of the triangle under the line $y=x$ for $x=[0,1]$, which is one half. The larger the Gini index, the larger the inequality of incomes. If $\gamma$ is the straight line $y=x$, then there would be complete equality with the Gini index being zero.

If $F$ is matrix-exponentially distributed we are able to provide explicit formulas for both the Lorenz curve and the Gini index.

Theorem 4.1. Let $F$ be the distribution function of a matrix-exponential distribution with representation $(\boldsymbol{\alpha}, \mathbf{S}, \mathbf{s})$, where $\mathbf{s}=-\mathbf{S e}$. Then the Lorenz curve is given by the formula

$$
\gamma: t \rightarrow\left(1-\boldsymbol{\alpha} e^{\mathbf{S} t} \mathbf{e}, 1-\frac{\boldsymbol{\alpha} \mathbf{S}^{-1}}{\boldsymbol{\alpha} \mathbf{S}^{-1} \mathbf{e}}\left(e^{\mathbf{S} t} \mathbf{e}+t e^{\mathbf{S} t} \mathbf{s}\right)\right)
$$

and the Gini index G by

$$
G=2\left(\boldsymbol{\alpha} \otimes \boldsymbol{\alpha}_{1}\right)\left(-\left(\mathbf{S} \oplus \mathbf{S}_{1}\right)\right)^{-1}(\mathbf{s} \otimes \mathbf{e})-1 .
$$

Proof. The Lorenz curve representation follows from Corollary 3.2 with $n=1$. The area $A$ under the curve $\gamma$ is given by

$$
\begin{aligned}
A & =\int_{0}^{\infty} F^{\prime}(t) F_{1}(t) d t \\
& =\int_{0}^{\infty} \boldsymbol{\alpha} e^{\mathbf{S} t} \mathbf{s}\left(1-\boldsymbol{\alpha}_{1} e^{\mathbf{S}_{1} t} \mathbf{e}\right) d t \\
& =1-\int_{0}^{\infty} \boldsymbol{\alpha} e^{\mathbf{S} t} \mathbf{s} \boldsymbol{\alpha}_{1} e^{\mathbf{S}_{1} t} \mathbf{e} d t \\
& =1+\left(\boldsymbol{\alpha} \otimes \boldsymbol{\alpha}_{1}\right)\left(\mathbf{S} \oplus \mathbf{S}_{1}\right)^{-1}(\mathbf{s} \otimes \mathbf{e}),
\end{aligned}
$$

where the last equality follows from Ref. ${ }^{[5]}$ and where $\otimes$ and $\oplus$ are the Kronecker product and sum respectively. The result now follows as $G=2\left(\frac{1}{2}-A\right)$. 

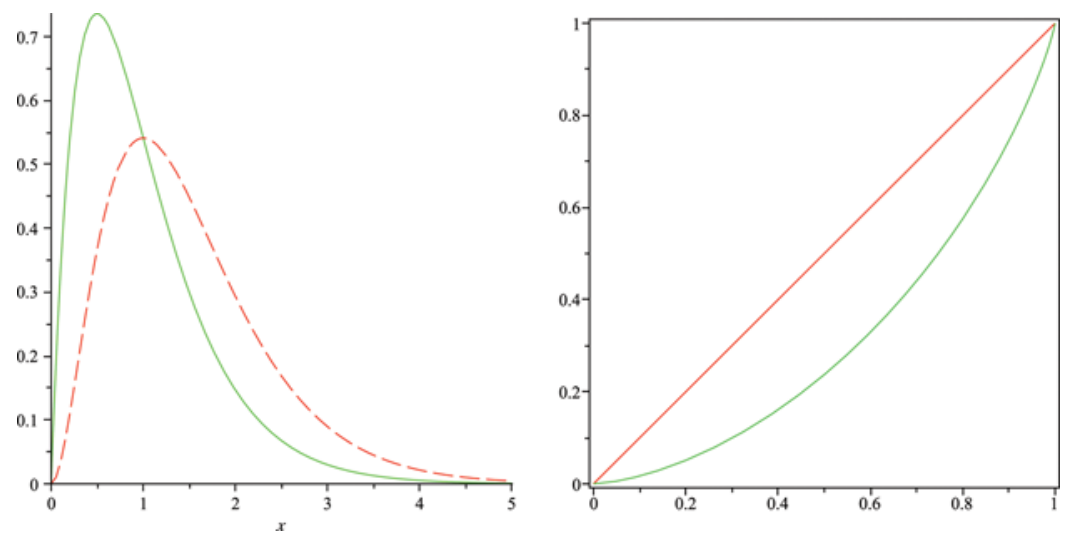

FIGURE 2 Left: Densities $f$ (solid) and its first order moment density $f_{1}$ (dashed). Right: Corresponding Lorenz curve. The Gini index is 0.3750 (color figure available online).
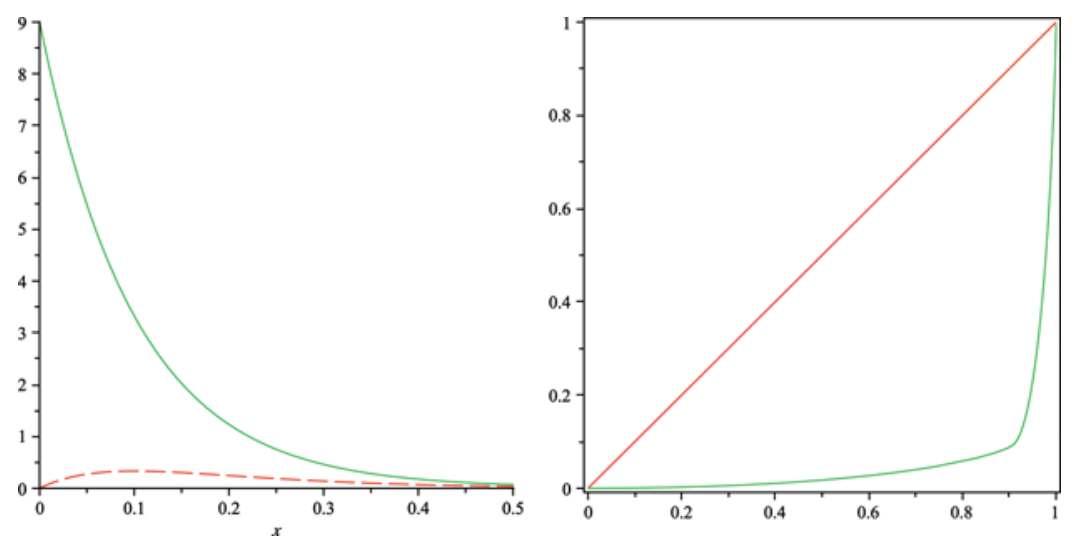

FIGURE 3 Left: Densities $g$ (solid) and its first order moment density $g_{1}$ (dashed). Right: Corresponding Lorenz curve. The Gini index is 0.8962 (color figure available online).
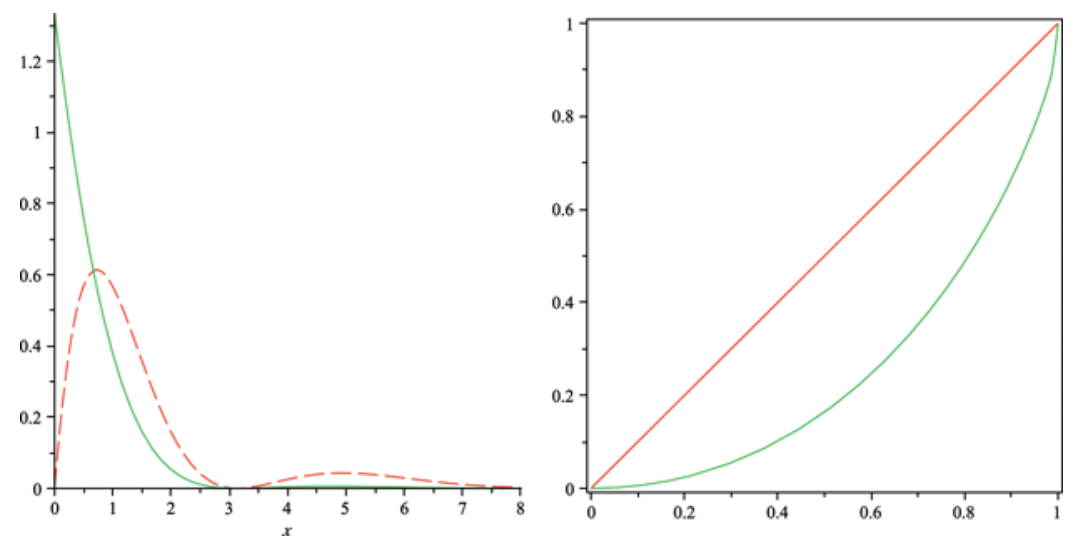

FIGURE 4 Left: Densities $h$ (solid) and its first order moment density $h_{1}$ (dashed). Right: Corresponding Lorenz curve. The Gini index is 0.4917 (color figure available online). 
Example 4.2. Consider the densities

$$
\begin{aligned}
& f(x)=4 x e^{-2 x}, \\
& g(x)=9 e^{-10 x}+\frac{1}{91} e^{-10 x / 91}, \\
& h(x)=\frac{2}{3} e^{-x}(1+\cos (x)) .
\end{aligned}
$$

The former two are phase-type with representations

$$
\left((1,0),\left(\begin{array}{cc}
-2 & 2 \\
0 & -2
\end{array}\right)\right), \quad\left(\left(\frac{9}{10}, \frac{1}{10}\right),\left(\begin{array}{cc}
-10 & 0 \\
0 & -\frac{10}{91}
\end{array}\right)\right)
$$

respectively while the latter is a matrix-exponential distribution not being phase-type with representation

$$
\left.(0,0,1),\left(\begin{array}{ccc}
-1 & 0 & 0 \\
-\frac{2}{3} & -1 & 1 \\
\frac{2}{3} & -1 & -1
\end{array}\right),\left(\begin{array}{c}
1 \\
\frac{2}{3} \\
\frac{4}{3}
\end{array}\right)\right) .
$$

The three cases are plotted in Figures 2, 3, and 4.

\section{ACKNOWLEDGMENTS}

The authors are grateful for the support by Otto Mønsteds foundation, The Danish research council for technology and production sciences grant no. 247-07-0090 and Sistema Nacional de Investigadores, Mexico. We would also like to the thank the anonymous referees for their very careful examination of the article, particularly as one referee pointed out an error in a previous formulation of Theorem 3.3.

Both authors have been working with and co-authored several papers with Marcel Neuts. Although this article is not directly related to these publications it certainly bears the spirit of Marcel's work. We would like to thank Marcel for his contributions to the field in general and in particular for the pleasure, joy, and inspiration it has been for us to work with him.

\section{REFERENCES}

1. Andersen, A.T.; Neuts, M.F.; Nielsen, B.F. PH-distributions arising through conditioning. Communications in Statistics: Stochastic Models 2000, 16 (1), 179-188.

2. Asmussen, S.; Bladt, M. Renewal theory and queueing algorithms for matrix- exponential distributions. In First International Conference on Matrix-Analytic Methods (MAM) in Stochastic Models; Alfa, A.; Chakravarthy, S.R., Eds.; 1997; 313-341. 
3. Bladt, M.; Neuts, M. Matrix-exponential distributions: Calculus and interpretations via flows. Stochastic Models 2003, 19 (1), 113-124.

4. Commault, C.; Chemla, J.P. On dual and minimal phase-type representations. Communications in Statistics: Stochastic Models 1993, 9 (3), 421-434.

5. Graham, A. Kronecker Products and Matrix Calculus with Applications; Ellis Horwood Series: London, 1981.

6. Kao, E.P.C.; Smith, M.S. On excess-, current-, and total-life distributions of phase-type renewal processes. Naval Research Logistics 1992, 39 (6), 789-799.

7. Neuts, M.F. Matrix-Geometric Solutions in Stochastic Models an Algorithmic Approach, Volume 2 of the John Hopkins Series in Mathematical Sciences; The John Hopkins University Press: Bethesda, MD, 1981.

8. Ramaswami, V. A duality theorem for the matrix paradigms in queueing theory. Communication in Statistical and Stochastic Models 1990, 6 (1), 151-161.

9. Sigman, K. Stationary Marked Point Processes: An Intuitive Approach; Chapman and Hall: New York, 1995.

10. Vaz, M.F.; Fortes, M.A. Grain size distribution: The lognormal and the gamma distributions functions. Metallurgica 1988, 22 35-40. 